\title{
STABILITY ANALYSIS OF GYROSCOPIC SYSTEMS WITH DISCRETE AND DISTRIBUTED DELAYS
}

\author{
Alexander Aleksandrov \\ Saint Petersburg State University \\ ITMO University \\ Russia \\ a.u.aleksandrov@spbu.ru
}

\author{
Aleksandr Semenov \\ ITMO University \\ Russia \\ sashkasem@mail.ru
}

\author{
Jingyuan Zhan \\ Beijing University of Technology \\ China \\ jyzhan@bjut.edu.cn
}

Article history:

Received 24.05.2019, Accepted 12.06.2019

\begin{abstract}
This paper provides stability analysis results for a linear mechanical system with a large parameter at the vector of gyroscopic forces and with delay in positional forces. Both cases of discrete and distributed delay are studied. Using the decomposition method and Lyapunov-Krasovskii functionals, conditions are found under which delay does not disturb the asymptotic stability of the considered system. The effectiveness of the obtained results is illustrated by a simulation example.
\end{abstract}

\section{Key words}

Gyroscopic system, delay, large parameter, Lyapunov direct method, asymptotic stability, decomposition, aggregation.

\section{Introduction}

The basic approach to the analysis of dynamics of complex and network systems is the decomposition method [Bullo, Cortes and Martinez, 2009; Frolov, Koronovskii, Makarov, Maksimenko, Goremyko and Hramov, 2017; Lakshmikantham, Leela and Martynyuk, 1989; Matrosov, 2001; Proskurnikov and Granichin, 2018; Siljak, 1991; Zubov, 1970]. The method is effectively used in various forms for the investigation of mechanical systems, see, for instance, [Andrievsky and Boikov, 2017; Alyshev, Dudarenko and Melnikov, 2018; Matrosov, 2001; Merkin, 1974] and the bibliography therein.

In [Zubov, 1970] and [Merkin, 1974], original approaches to the decomposition of linear time-invariant mechanical systems with a large parameter at the vectors of velocity and gyroscopic forces, respectively, have been proposed. The results of [Zubov, 1970] and
[Merkin, 1974] permit us to reduce the stability problem for a considered second order system to that for two independent first order subsystems.

These approaches have got further development in [Kosov, 2005; Aleksandrov, Chen, Kosov and Zhang, 2011; Aleksandrov, Kosov and Chen, 2011; Aleksandrov and Aleksandrova, 2016], and new stability conditions were obtained not only for linear time-invariant systems, but also for systems with nonlinear and nonstationary force fields.

In [Kuptsov, 2000; Aleksandrov, Aleksandrova and Zhabko, 2014], such approaches were applied to the stability analysis of linear gyroscopic systems with a large parameter at the vector of gyroscopic forces and with delay in positional forces. It was proved that if auxiliary first order delay-free subsystems are asymptotically stable, then, for sufficiently large values of the parameter, one can guarantee asymptotic stability of an original second order time-delay system. However, it should be noted that the results of [Kuptsov, 2000; Aleksandrov, Aleksandrova and Zhabko, 2014] are delay-dependent. At the same time, in numerous applications, delay-independent stability conditions are required, see [Gu, Kharitonov and Chen, 2003].

In this paper, we propose another approach to the justification of possibility of decomposition for linear gyroscopic systems with time delay that permits to derive delay-independent stability conditions. In addition, we will show that the approach can be used for the stability investigation of gyroscopic systems with distributed delay, as well.

\section{Statement of the Problem}

Let motions of a mechanical system be described by the equations

$$
A \ddot{x}(t)+(B+h G) \dot{x}(t)+C x(t)+D x(t-\tau)=0 .
$$


Here $x(t), \dot{x}(t) \in \mathbb{R}^{n}$ are vectors of generalized coordinates and velocities, respectively; $A, B, G, C, D$ are constant matrices; $h$ is a positive parameter; $\tau$ is a constant nonnegative delay.

We assume that matrices $A$ and $B$ are symmetric and positive definite, and matrix $G$ is skew-symmetric. Thus, the considered mechanical system is influenced by the dissipative forces $-B \dot{x}(t)$, gyroscopic forces $-h G \dot{x}(t)$ and positional forces $-C x(t)-D x(t-\tau)$.

Equations of the form (1) are widely used as linear approximations of models of gyroscopic systems (see [Merkin, 1974; Zubov, 1970]). As examples of such models, a gyrovertic with radial correction and force gyroscopic horizon with delay in feedback laws may be considered [Merkin, 1974]. In these systems, the parameter $h$ can be treated as a frequency of a gyroscope rotation.

Let initial functions for solutions of (1) belong to the space $C^{1}\left([-\tau, 0], \mathbb{R}^{n}\right)$ of continuously differentiable functions $\varphi(\theta):[-\tau, 0] \rightarrow \mathbb{R}^{n}$ with the uniform norm

$$
\|\varphi\|_{\tau}=\max _{\theta \in[-\tau, 0]}(\|\varphi(\theta)\|+\|\dot{\varphi}(\theta)\|) .
$$

Here $\|\cdot\|$ denotes the Euclidean norm of a vector. Let $x_{t}$ stand for the restriction of a solution $x(t)$ of (1) to the segment $[t-\tau, t]$, i.e., $x_{t}: \theta \rightarrow x(t+\theta), \theta \in[-\tau, 0]$. In what follows we assume that $n$ is an even number and $\operatorname{det} G \neq 0$.

We will look for asymptotic stability conditions for the system (1).

In [Kuptsov, 2000; Aleksandrov, Aleksandrova and Zhabko, 2014] it was proved that if the auxiliary delayfree subsystem

$$
G \dot{y}(t)+(C+D) y(t)=0
$$

is asymptotically stable, then, for every $\tau \geq 0$, there exists $h_{0}>0$ such that the system (1) is asymptotically stable for any $h \geq h_{0}$. To derive such a result, in [Kuptsov, 2000], the first Lyapunov method was used, whereas, in [Aleksandrov, Aleksandrova and Zhabko, 2014], the Lyapunov direct method and the Razumikhin approach were applied. However, it should be noted that lower bounds for admissible values of parameter $h$ obtained in [Kuptsov, 2000] and [Aleksandrov, Aleksandrova and Zhabko, 2014] depend on the magnitude of delay.

In the present paper, we will use another approach to the stability analysis of the system (1) that permits us to derive delay-independent asymptotic stability conditions. The approach is based on the decomposition method and a special construction of LyapunovKrasovskii functional for (1).

In addition, we will show that with the aid of the approach, sufficient conditions of asymptotic stability can be obtained for linear gyroscopic systems with distributed delay.

\section{A System with Discrete Delay}

Instead of (2), construct the auxiliary time-delay subsystem

$$
h G \dot{y}(t)+C y(t)+D y(t-\tau)=0 .
$$

Denote $M=-G^{-1} C, N=-G^{-1} D$.

Theorem 1. Assume that there exist constant symmetric positive definite matrices $P$ and $Q$ such that the matrix

$$
\left(\begin{array}{cc}
M^{\top} P+P M+Q & P N \\
N^{\top} P & -Q
\end{array}\right)
$$

is negative definite. Then one can choose a number $h_{0}>0$ such that if $h \geq h_{0}$, then the system (1) is asymptotically stable for any nonnegative delay $\tau$.

\section{Proof. Let}

$$
y(t)=x(t)+(B+h G)^{-1} A \dot{x}(t), \quad z(t)=\dot{x}(t) .
$$

The substitution (4) transforms (1) to the system

$$
\begin{array}{r}
\dot{y}(t)=\frac{1}{h} M y(t)+\frac{1}{h} N y(t-\tau) \\
+\frac{1}{h}(B+h G)^{-1} B G^{-1}(C y(t)+D y(t-\tau)) \\
+(B+h G)^{-1} C(B+h G)^{-1} A z(t) \\
+(B+h G)^{-1} D(B+h G)^{-1} A z(t-\tau), \\
A \dot{z}(t)=-(B+h G) z(t)-C y(t) \\
+C(B+h G)^{-1} A z(t)-D y(t-\tau) \\
+D(B+h G)^{-1} A z(t-\tau) .
\end{array}
$$

The system (5) can be treated as a complex system describing interaction of the subsystem (3) and the subsystem

$$
A \dot{z}(t)=-(B+h G) z(t) .
$$

It is known [Gu, Kharitonov and Chen, 2003], that, under the conditions of Theorem 1, the subsystem (3) is asymptotically stable for any $\tau \geq 0$, and a LyapunovKrasovskii functional for (3) can be chosen in the form

$$
V_{1}\left(y_{t}\right)=h y^{\top}(t) P y(t)+\int_{t-\tau}^{t} y^{\top}(s) Q y(s) d s .
$$


Moreover, the subsystem (6) is asymptotically stable and admits the Lyapunov function

$$
V_{2}(z)=\frac{1}{2} z^{\top} A z
$$

Let

$V\left(y_{t}, z_{t}\right)=V_{1}\left(y_{t}\right)+\frac{1}{h} V_{2}(z(t))+\frac{\lambda}{h} \int_{t-\tau}^{t}\|z(s)\|^{2} d s$,

where $\lambda$ is a positive parameter, and $V_{1}\left(y_{t}\right), V_{2}(z)$ are defined by the formulae (7) and (8), respectively. Then

$$
\begin{gathered}
a_{1}\left(h\|y(t)\|^{2}+\frac{1}{h}\|z(t)\|^{2}\right) \leq V\left(y_{t}, z_{t}\right) \\
\leq a_{2}\left(h\|y(t)\|^{2}+\frac{1}{h}\|z(t)\|^{2}\right. \\
\left.+\int_{t-\tau}^{t}\|y(s)\|^{2} d s+\frac{\lambda}{h} \int_{t-\tau}^{t}\|z(s)\|^{2} d s\right), \\
\left.+B G^{-1} D y(t-\tau)\right)+h C(B+h G)^{-1} A z(t) \\
+2 y^{\top}(t) P(B+h G)^{-1}\left(B G^{-1} C y(t)\right. \\
+\frac{1}{h} z^{\top}(t) B z(t)+\frac{\lambda}{h}\|z(t)\|^{2}-\frac{\lambda}{h}\|z(t-\tau)\|^{2} \\
+y^{\top}(t) Q y(t)-2 y^{\top}(t-\tau) Q y(t-\tau)+2 y^{\top}(t) P N y(t-\tau)
\end{gathered}
$$

$$
+\frac{1}{h} z^{\top}(t)\left(-C y(t)+C(B+h G)^{-1} A z(t)\right.
$$

$$
\left.-D y(t-\tau)+D(B+h G)^{-1} A z(t-\tau)\right)
$$

$$
\leq-a_{3}\left(\|y(t)\|^{2}+\|y(t-\tau)\|^{2}\right)-\frac{1}{h}\left(a_{4}-\lambda\right)\|z(t)\|^{2}
$$

$$
\begin{gathered}
+\frac{a_{5}\|y(t)\|}{h}(\|y(t)\|+\|y(t-\tau)\|+\|z(t)\|+\|z(t-\tau)\|) \\
+\frac{a_{6}\|z(t)\|}{h}\left(\|y(t)\|+\|y(t-\tau)\|+\frac{1}{h}\|z(t)\|\right. \\
\left.+\frac{1}{h}\|z(t-\tau)\|\right)-\frac{\lambda}{h}\|z(t-\tau)\|^{2} .
\end{gathered}
$$

Here $a_{1}, a_{2}, a_{3}, a_{4}, a_{5}, a_{6}$ are positive constants independent of $h, \lambda$ and $\tau$.

Let $\lambda=1 / \sqrt{h}$. Then there exists $h_{0}>0$ such that

$$
\begin{gathered}
\left.\dot{V}\right|_{(5)} \leq-\frac{a_{3}}{2}\left(\|y(t)\|^{2}+\|y(t-\tau)\|^{2}\right) \\
-\frac{a_{4}}{2 h}\|z(t)\|^{2}-\frac{\lambda}{2 h}\|z(t-\tau)\|^{2}
\end{gathered}
$$

for $h \geq h_{0}$. Hence [Gu, Kharitonov and Chen, 2003], the system (5) is asymptotically stable for $h \geq h_{0}$ and for any value of delay. Then, from the properties of the transformation (4), it follows delay-independent asymptotic stability of (1). This completes the proof.

\section{A System with Distributed Delay}

Next, consider the system with distributed delay

$$
\begin{array}{r}
A \ddot{x}(t)+(B+h G) \dot{x}(t)+C x(t) \\
+D \int_{t-\tau}^{t} x(s) d s=0 .
\end{array}
$$

Here all notations are the same as for (1). 
It is worth noting that systems with distributed delays arise in traffic flow models, in network control systems, in PID controller design and in other engineering applications [Bullo, Cortes and Martinez, 2009; Gu, Kharitonov and Chen, 2003; Fridman, 2014; Solomon and Fridman, 2013]. Some conditions of stability and stabilization of mechanical systems with distributed delays were obtained, for instance, in [Anan'evskii and Kolmanovskii, 1989; Pavlikov, 2007].

Let us show that the approach proposed in the previous section can be used for the stability analysis of the system (9), as well.

In this case, we consider the following auxiliary subsystem:

$$
h G \dot{y}(t)+C y(t)+D \int_{t-\tau}^{t} y(s) d s=0 .
$$

Denote $M=-G^{-1} C, N=-G^{-1} D$.

Theorem 2. Assume that, for a given $\tau \geq 0$, there exist constant symmetric positive definite matrices $P$ and $Q$ such that the matrix

$$
\left(\begin{array}{cc}
\frac{1}{\tau}\left(M^{\top} P+P M\right)+Q & P N \\
N^{\top} P & -Q
\end{array}\right)
$$

is negative definite. Then one can choose a number $h_{0}>0$ such that if $h \geq h_{0}$, then the system (9) is asymptotically stable.

Proof. Using the substitution (4), we arrive at the system

$$
\begin{array}{r}
\dot{y}(t)=\frac{1}{h} M y(t)+\frac{1}{h} N y(t-\tau) \\
+\frac{1}{h}(B+h G)^{-1} B G^{-1} C y(t) \\
+\frac{1}{h}(B+h G)^{-1} B G^{-1} D \int_{t-\tau}^{t} y(s) d s \\
+(B+h G)^{-1} C(B+h G)^{-1} A z(t) \\
+(B+h G)^{-1} D(B+h G)^{-1} A \int_{t-\tau}^{t} z(s) d s, \\
A \dot{z}(t)=-(B+h G) z(t)-C y(t) \\
+C(B+h G)^{-1} A z(t)-D \int_{t-\tau}^{t} y(s) d s \\
+D(B+h G)^{-1} A \int_{t-\tau}^{t} z(s) d s .
\end{array}
$$

From the conditions of Theorem 2 it follows (see [Gu, Kharitonov and Chen, 2003; Fridman, 2014]) that the subsystem (10) is asymptotically stable and admits the
Lyapunov-Krasovskii functional

$$
\begin{array}{r}
\widetilde{V}_{1}\left(y_{t}\right)=h y^{\top}(t) P y(t) \\
+\int_{-\tau}^{0} \int_{t+\theta}^{t} y^{\top}(s) Q y(s) d s d \theta .
\end{array}
$$

Choose a candidate Lyapunov-Krasovskii functional for (11) in the form

$$
\begin{gathered}
\widetilde{V}\left(y_{t}, z_{t}\right)=\widetilde{V}_{1}\left(y_{t}\right)+\frac{1}{h} V_{2}(z(t)) \\
+\frac{\lambda}{h} \int_{t-\tau}^{t}(s+\tau-t)\|z(s)\|^{2} d s,
\end{gathered}
$$

where $\lambda$ is a positive parameter, and $\widetilde{V}_{1}\left(y_{t}\right), V_{2}(z)$ are defined by the formulae (12) and (8), respectively. We obtain

$$
a_{1}\left(h\|y(t)\|^{2}+\frac{1}{h}\|z(t)\|^{2}\right) \leq \widetilde{V}\left(y_{t}, z_{t}\right)
$$

$$
\leq a_{2}\left(h\|y(t)\|^{2}+\frac{1}{h}\|z(t)\|^{2}\right.
$$

$$
\left.+\int_{-\tau}^{0} \int_{t+\theta}^{t}\|y(s)\|^{2} d s+\frac{\lambda}{h} \int_{t-\tau}^{t}\|z(s)\|^{2} d s\right)
$$

$$
\begin{gathered}
\left.\dot{\tilde{V}}\right|_{(11)} \leq-a_{3}\left(\|y(t)\|^{2}+\int_{t-\tau}^{t}\|y(s)\|^{2} d s\right) \\
-\frac{1}{h}\left(a_{4}-\lambda \tau\right)\|z(t)\|^{2}-\frac{\lambda}{h} \int_{t-\tau}^{t}\|z(s)\|^{2} d s \\
+\frac{a_{5}\|y(t)\|}{h}\left(\|y(t)\|+\int_{t-\tau}^{t}\|y(s)\| d s\right.
\end{gathered}
$$

$$
\left.+\|z(t)\|+\int_{t-\tau}^{t}\|z(s)\| d s\right)
$$




$$
\begin{gathered}
+\frac{a_{6}\|z(t)\|}{h}\left(\|y(t)\|+\int_{t-\tau}^{t}\|y(s)\| d s\right. \\
\left.+\frac{1}{h}\|z(t)\|+\frac{1}{h} \int_{t-\tau}^{t}\|z(s)\| d s\right) .
\end{gathered}
$$

Here $a_{1}, a_{2}, a_{3}, a_{4}, a_{5}, a_{6}$ are positive constants independent of $h$ and $\lambda$.

If $\lambda=1 / \sqrt{h}$, then the estimate

$$
\begin{gathered}
\left.\dot{\tilde{V}}\right|_{(11)} \leq-\frac{a_{3}}{2}\left(\|y(t)\|^{2}+\int_{t-\tau}^{t}\|y(s)\|^{2} d s\right) \\
-\frac{a_{4}}{2 h}\|z(t)\|^{2}-\frac{\lambda}{2 h} \int_{t-\tau}^{t}\|z(s)\|^{2} d s
\end{gathered}
$$

holds for sufficiently large values of $h$. This completes the proof.

Remark 1. Unlike Theorem 1, Theorem 2 provides us delay-dependent asymptotic stability conditions.

\section{Results of a Numerical Simulation}

To illustrate the effectiveness of the obtained results, consider the system

$$
\begin{gathered}
\ddot{x}(t)+\left(\left(\begin{array}{ll}
2 & 1 \\
1 & 1
\end{array}\right)+h\left(\begin{array}{ll}
0 & -1 \\
1 & 0
\end{array}\right)\right) \dot{x}(t) \\
+\left(\begin{array}{ll}
1 & -4 \\
2 & -1
\end{array}\right) x(t)-\left(\begin{array}{ll}
0 & 0 \\
1 & 2
\end{array}\right) x(t-\tau)=0 .
\end{gathered}
$$

Here $x(t), \dot{x}(t) \in \mathbb{R}^{2} ; h$ is a positive parameter; $\tau$ is a nonnegative delay.

Construct the auxiliary subsystem (3) for (13). We obtain

$$
h \dot{y}(t)=\left(\begin{array}{rl}
-2 & 1 \\
1 & -4
\end{array}\right) y(t)+\left(\begin{array}{ll}
1 & 2 \\
0 & 0
\end{array}\right) y(t-\tau) .
$$

With the aid of the results of [Aleksandrov and Mason, 2016], it can be proved that the subsystem (14) admits a Lyapunov-Krasovskii functional of the form (7). Hence (see Theorem 1), there exists a number $h_{0}>0$ such that if $h \geq h_{0}$, then the system (13) is asymptotically stable for any nonnegative delay $\tau$.

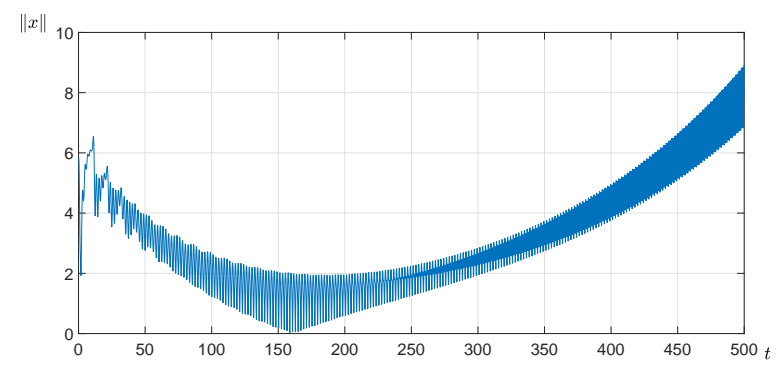

Figure 1. The results of simulation for $h=3.3$

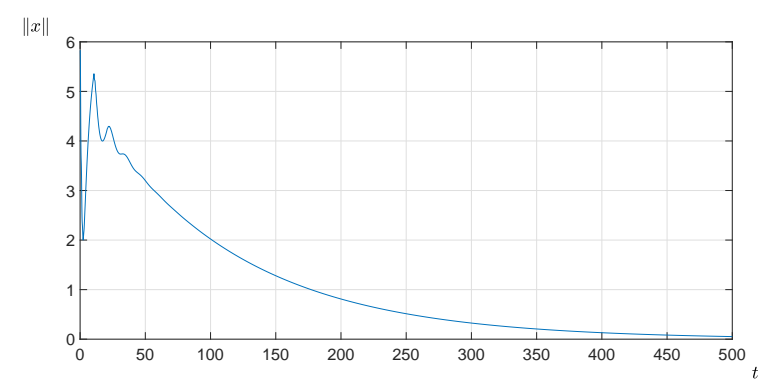

Figure 2. The results of simulation for $h=8$

For simulation, we choose $\tau=10$ and $x(t)=$ $(-3,5)^{\top}$ for $t \in[-10,0]$. In Figs. 1 and 2 the dependence of $\|x\|$ on $t$ is presented.

First, the case is considered where $h=3.3$. Figure 1 shows that, for this value of $h$, the system (13) is unstable.

Next, we take $h=8$ and repeat calculations with the same delay and initial conditions. Figure 2 demonstrates the asymptotic stability of the corresponding system.

\section{Conclusion}

In this contribution, with the aid of the decomposition method and special constructions of LyapunovKrasovskii functionals, sufficient conditions of the asymptotic stability are found for linear gyroscopic systems with discrete and distributed delays. In is worth noting that, for the case of discrete delay, these conditions are delay-independent, whereas, for systems with distributed delay, admissible values of parameter $h$ depend on delay.

We outline some suggestions for further research. First, using the constructed Lyapunov-Krasovskii functionals, estimates of the convergence rate of solutions can be derived for considered systems. Next, the developed approaches can be applied to the stability analysis of mechanical systems with switched force fields. In addition, we believe the approaches in this paper can be useful for the study the consensus problem for multi-agent systems described by double integrators with communication delays. 


\section{Acknowledgements}

This work was supported in part by Government of Russian Federation (Grant no. 08-08), by the Russian Foundation for Basic Research (Grant no. 19-0100146-a), by the National Natural Science Foundation of China (Grant no. 61803007) and by the Rail Transit Joint Funds of Beijing Natural Science Foundation and Traffic Control Technology (Grant no. L171001).

\section{References}

Aleksandrov, A.Yu., and Aleksandrova, E.B. (2016) Asymptotic stability conditions for a class of hybrid mechanical systems with switched nonlinear positional forces. Nonlinear Dynamics, 83(4), pp. 24272434.

Aleksandrov, A.Yu., Aleksandrova, E.B., and Zhabko, A.P. (2014) Asymptotic stability conditions for certain classes of mechanical systems with time delay. WSEAS Transactions on Systems and Control, 9 , pp. 388-397.

Aleksandrov, A.Yu., Chen, Y., Kosov, A.A., and Zhang, L. (2011) Stability of hybrid mechanical systems with switching linear force fields. Nonlinear Dyn. Syst. Theory, 11(1), pp. 53-64.

Aleksandrov, A.Yu., Kosov, A.A., and Chen, Y. (2011) Stability and stabilization of mechanical systems with switching. Automation and Remote Control, 72(6), pp. 1143-1154.

Aleksandrov, A., and Mason, O. (2016) Diagonal Riccati stability and applications. Linear Algebra and its Appl., 492, pp. 38-51.

Alyshev, A., Dudarenko, N., and Melnikov, V. (2018) Parametric identification of reaction wheel pendulums with adaptive control. Cybernetics and Physics, 7(2), pp. 57-65.

Anan'evskii, I.M., and Kolmanovskii, V.B. (1989) On stabilization of some control systems with an after-effect. Automation and Remote Control, (9), pp. 1174-1181.

Andrievsky, B., and Boikov, V. (2017) Experimental study of multiresonance mechatronic vibrational laboratory set-up. Cybernetics and Physics, 6(1), pp. 512.
Bullo, F., Cortes, J., and Martinez, S. (2009) Distributed Control of Robotics Networks. Princeton Univ. Press. Princeton.

Fridman, E. (2014) Tutorial on Lyapunov-based methods for time-delay systems. European J. of Control, 20, pp. 271-283.

Frolov, N., Koronovskii, A, Makarov, V., Maksimenko, V., Goremyko, M., and Hramov, A. (2017) Control of pattern formation in complex networks by multiplexity. Cybernetics and Physics, 6(3), pp. 121125.

Gu, K., Kharitonov, V.L., and Chen, J. (2003) Stability of Time-delay Systems. Birkhauser, Boston, MA.

Kosov, A.A. (2005) Stability investigation of singular systems by means of the Lyapunov vector functions method. Vestnik St. Petersburg University, Ser. 10, (4), pp. 123-129. (in Russian)

Kuptsov, S.Yu. (2000) Stability of a gyroscopic system with regard to delays. In Proc. 31th Conf. Processes of Control and Stability. St. Petersburg, Russia, April 1-7, pp. 86-89. (in Russian).

Lakshmikantham, V., Leela, S., and Martynyuk, A.A. (1989) Stability Analysis of Nonlinear Systems. Marcel Dekker, New York.

Matrosov, V.M. (2001) The Method of Vector Lyapunov Functions: Analysis of Dynamical Properties of Nonlinear Systems. Fizmatlit, Moscow. (in Russian).

Merkin, D.R. (1974) Gyroscopic Systems. Nauka, Moscow. (in Russian).

Pavlikov, S.V. (2007) Stabilization of the motions of controlled mechanical systems by a regulator with delay. Doklady Mathematics, 75(1), pp. 174-176.

Proskurnikov, A., and Granichin, O. (2018) Evolution of clusters in large-scale dynamical networks. Cybernetics and Physics, 7(3), pp. 102-129.

Siljak, D.D. (1991) Decentralized Control of Complex Systems. Academic Press, Boston.

Solomon, O., and Fridman, E. (2013) New stability conditions for systems with distributed delays. Automatica, 49, pp. 3467-3475.

Zubov, V.I. (1970) Analytical Dynamics of Gyroscopic Systems. Sudostroenie, Leningrad. (in Russian). 